\section{Controllability Distributions and Systems Approximations: a Geometric Approach}

\author{
A. C. Ruiz and H. Nijmeijer
}

\begin{abstract}
Given a nonlinear system we determine a relation at an equilibrium between controllability distributions defined for a nonlinear system and a Taylor series approximation of it. The value of such a relation is appreciated if we recall that the solvability conditions as well as the solutions to some control synthesis problems can be stated in terms of geometric concepts like controlled invariant (controllability) distributions. The relation between these distributions at the equilibrium will help us to decide when the solvability conditions of this kind of problems are equivalent for the nonlinear system and its approximation. Some examples illustrate the results.
\end{abstract}

\section{INTRODUCTION}

For decades it has been the common practice of control engineers to solve nonlinear control synthesis problems by using a linear approximation of the nonlinear system around an operating point and after application of linear control techniques, use the resulting linear solution as a linear approximation of a true solution for the original nonlinear control problem, see e.g. [14], [15], [17], and other applications. That this approach is in some cases successful for specific control objectives like input-output decoupling, model matching, etc., is partially understood, see e.g., [2], [4], [19], [10], [11], [18]. It is not a general rule, however, that the linearization procedure is always justified, even in the case when a particular nonlinear control problem is solvable for the nonlinear system and for the linearization the solutions of the linear problem do not necessarily act as a first order approximation of a solution for the nonlinear problem, as is stated as a principle in [13, pp. 5].

In nonlinear control theory, differential geometric concepts as controlled invariant and controllability distributions play a fundamental role in the solution of synthesis problems like disturbance decoupling, input-output decoupling, etc., see [8], [5], [3]. Not only the solvability conditions of this kind of problems can be stated in terms of these distributions, but also these distributions are fundamental to characterize all solutions for a particular control problem, see e.g., [8], [3], [5] and [1] in a linear context. Therefore, it is of interest for a relation at the equilibrium between these distributions defined for a given nonlinear system and an approximation of it.

We consider nonlinear analytic control systems of the form

$$
\Sigma\left\{\begin{array}{l}
\dot{x}=f(x)+\sum_{i=1}^{m} g_{i}(x) u_{i}=f(x)+g(x) u, \\
y_{i}=h_{i}(x), \quad i \in \underline{p}=\{1, \cdots, p\}
\end{array}\right.
$$

defined on an open neighborhood $M$ of $x_{0}$ in $\mathbb{R}^{n}$, where $x_{0}$ is an equilibrium point for $\Sigma$, i.e., $f\left(x_{0}\right)=0$ and $h\left(x_{0}\right)=0, h:=$ $\left(h_{1}, \cdots, h_{p}\right)^{T}$. Without loss of generality we assume throughout $x_{0}=0$. We approximate the system $\Sigma$ and thus the vector fields $f, g_{1}, \cdots, g_{m}$, and the output functions $h_{1}, \cdots, h_{p}$, by means of a Taylor series expansion of $f, g_{1}, \cdots, g_{m}, h_{1}, \cdots, h_{p}$, respectively, around the equilibrium point 0 . Regarding $\Sigma$ as a system locally defined about 0 we denote the $k$ th order approximation of $\Sigma$ as

$$
\Sigma^{k}\left\{\begin{array}{l}
\dot{x}=f^{k}(x)+\sum_{i=1}^{m} g_{i}^{k-1}(x) u_{i}=f^{k}(x)+g^{k-1}(x) u \\
y_{i}^{k}=h_{i}^{k}(x), \quad i \in \underline{p}
\end{array}\right.
$$

Manuscript received October 14, 1992; revised May 15, 1993. This work was supported in part by CONACYT, Mexico.

The authors are with the Department of Applied Mathematics, University of Twente, The Netherlands.

IEEE Log Number 9401669 with $f^{k}, h^{k}$, the $k$ th order approximation of $f, h$ respectively, about the equilibrium and $g_{i}^{k-1}$ is the $(k-1)$ th order Taylor series of $g_{i}$ about 0 . Clearly, $\Sigma^{k}$ is defined on the same state space as $\Sigma$.

In [6], a nonlinear change of coordinates and feedback are used to construct linear approximations that are accurate to higher orders. Our analysis follows basically the same philosophy of [11] where controlled invariant distributions defined for $\Sigma^{k}$ and $\Sigma^{k+1}$ were studied.

The main result of the paper is contained in Section IV. In Section II we fix our notation and recall some basic definitions. Section III deals with accessibility distributions defined for $\Sigma$ and $\Sigma^{k}$. Finally, conclusions are drawn in Section V.

\section{PRELIMINARIES}

The interested reader can find the definitions and geometric notions introduced in this section in [5], [8]. With respect to the nonlinear systems $\Sigma$ and $\Sigma^{k}$, we impose throughout the following conditions on the input vector fields $g,\left(g^{k-1}\right)$ and the output maps $h\left(h^{k}\right)$. Define $G:=\operatorname{span}\left\{g_{1}, \cdots, g_{m}\right\}, G^{k}:=\operatorname{span}\left\{g_{1}^{k-1}, \cdots, g_{m}^{k-1}\right\}$ and $d h, d h^{k}$ the codistributions span $\left\{d h_{1}, \cdots, d h_{p}\right\}$ and span $\left\{d h_{1}^{k}, \cdots, d h_{p}^{k}\right\}$, respectively.

Assumption 1: Consider the systems $\Sigma$ and $\Sigma^{k}, k \geq 1$. Assume that

1) $\operatorname{dim} G=\operatorname{dim} G^{k}=m$ on $M$.

2) $\operatorname{dim} d h=\operatorname{dim} d h^{k}=m$ on $M$.

Basic in the sequel are the following concepts. A distribution $\Delta$ is involutive if $[X, Y] \in \Delta$ for all vector fields $X, Y \in \Delta$. A constant dimensional involutive distribution $\Delta$ is said to be controlled invariant if there exists a $C^{\omega}$ regular static state feedback

$$
u=\alpha(x)+\beta(x) v
$$

with $\alpha: M \rightarrow \mathbb{R}^{m}, \beta: M \rightarrow \mathbb{R}^{m \times m}, \beta(x)$ a nonsingular matrix for all $x \in M$ and $v \in \mathbb{R}^{m}$ such that after applying (1) to $\Sigma$ the modified vector fields $\tilde{f}:=f+g \alpha, \tilde{g}_{i}:=(g \beta)_{i}, i \in \underline{m}$, satisfy $[\tilde{f}, X] \subset \Delta,\left[\tilde{g}_{i}, X\right] \subset \Delta, i \in \underline{m}$, for all $X \in \Delta$.

The accessibility distribution $\bar{C}$ of $\Sigma$ is the smallest involutive distribution invariant under $f$ which contains $G$. A controllability distribution $D$ is an involutive distribution that contains a distribution $\hat{G} \subset G$ that is invariant under $\tilde{f}$ and $\tilde{g}_{i}, i \in \underline{m}$ for some feedback (1) and $D$ is the smallest distribution containing $G$ having this property.

Denote by $\Delta^{*}\left(\Delta_{k}^{*}\right)\left(\Pi^{*}\left(\Pi_{k}^{*}\right)\right)$, the maximal controlled invariant distribution (maximal controllability distribution) contained in $\operatorname{ker} d h\left(\operatorname{ker} d h^{k}\right)$ of $\Sigma\left(\Sigma^{k}\right)$, respectively. An algorithm to compute $\Pi_{k}^{*}\left(\Pi^{*}\right)$ is taken from [5].

Algorithm:

$$
\begin{gathered}
\Pi_{0}^{k}=\Delta_{k}^{*} \bigcap G^{k}, \\
\Pi_{\mu+1}^{k}=\Delta_{k}^{*} \bigcap\left(\left[f, \Pi_{\mu}^{k}\right]+\sum_{i=1}^{m}\left[g_{i}, \Pi_{\mu}^{k}\right]+G^{k}\right), \quad \mu \geq 0 .
\end{gathered}
$$

The algorithm to compute $\Pi^{*}$ is obtained by replacing $\Pi_{i}^{k},\left(\Delta_{k}^{*}, G^{k}\right), i \geq 0$, by $\Pi_{i},\left(\Delta^{*}, G\right)$, respectively. In connection with this algorithm we make some standard assumptions.

Assumption 2:

1) For each $k \geq 1$, there exists an integer $k^{*}\left(k^{*}(k)\right) \geq 0$ such that the algorithm terminates in the $k^{*}\left(k^{*}(k)\right)$ th step when applied to $\Sigma\left(\Sigma^{k}\right)$.

2) $\Pi_{i}, \Pi_{i}^{k}, i \in \mathbb{N}, k \geq 1$, have constant dimension on $M$.

3) The distributions $\Delta^{*}, \Delta_{k}^{*}, k \geq 1$, have constant dimension on $M$. 
Remark 2.1: The algorithm provides a sequence of nondecreasing distributions and given Assumption 2-1) it terminates whenever $\Pi_{\mu+1}=\Pi_{\mu}=\Pi^{*}$, for some $\mu \geq 0$, [5]. Note that $\Pi^{*}$ generated by the algorithm is, in general, not an involutive distribution. Its involutivity is assured, however, by the constant rank conditions in Assumption 2. Moreover, since dim ker dh $=n-p$ and thus dim $\Delta^{*} \leq n-p$ then $\operatorname{dim} \Pi^{*} \leq n-p$.

Actually, $C$ associated to $\Sigma$ can be computed by applying the algorithm and replacing $\Delta^{*}$ by $T M . C$ is usually called the strong accessibility distribution of $\Sigma$. We investigate in which cases does there exist a relation between $C(x)$ and $C^{k}(x)$ at the equilibrium. The relevance of knowing such a relation is better appreciated if we recall that the system $\Sigma$ is locally strong accessible about 0 if and only if $\operatorname{dim} C(0)=n,[16]$. Therefore a relation between $C(0)$ and $C^{k}(0)$ can be useful to recognize accessibility properties of $\Sigma$ by a hopefully simpler analysis of $\Sigma^{k}$.

\section{ACCESSIBILITY DISTRIBUTIONS FOR $\Sigma$ AND $\Sigma^{k}$}

To give some insight into the problem we start by studying the accessibility distribution defined for $\Sigma$ and its first order approximation $\Sigma^{1}$. The first order approximation of $\Sigma$ is given by

$$
\Sigma^{1}: \quad \dot{z}=A z+B \tilde{u}, \quad \tilde{y}=C z
$$

with $A:=(\partial f / \partial x)(0), B:=\left[b_{1}, \cdots, b_{m}\right], b_{i}:=g_{i}(0), C:=$ $(\partial h / \partial x)(0), \tilde{u} \in \mathbf{R}^{m}$ and $\tilde{y} \in \mathbf{R}^{p}$. The distribution $C^{1}(z)$ in this case reduces to the well known controllable subspace

$$
R=\langle A \mid \operatorname{Im} B\rangle=\operatorname{span}\left\{B, A B, \cdots, A^{n-1} B\right\} .
$$

Our interest is to compare the controllability subspace $R$ defined for $\Sigma^{1}$ with the accessibility distribution $C$ associated with $\Sigma$, at the equilibrium. To do so, we may consider the linear subspace $R$ as a flat distribution on $T M$. In general, $C^{1}(0) \subseteq C(0)$. For the second order approximation of $\Sigma$, denoted $\Sigma^{2}$, we intuitively expect that $C^{2}(0) \subseteq C(0)$. It turns out, however, that this is not always the case. An example of this phenomenon is in order. For this we introduce some extra notation.

Let $C_{i}^{k}, i=0,1, \cdots$, be a set of distributions associated with $\Sigma^{k}$ defined as

$$
\begin{gathered}
C_{0}^{k}(x)=\operatorname{span}\left\{g_{1}^{k-1}, \cdots, g_{m}^{k-1}\right\}(x) \\
C_{i}^{k}(x)=\operatorname{span}\left\{\left[X_{i},\left[\cdots,\left[X_{1}, g_{j}^{k-1}\right] \cdots\right]\right](x)\right. \\
\left.+\sum_{l=0}^{i-1} C_{l}^{k}(x), j \in \underline{m}\right\}
\end{gathered}
$$

for $i=1,2, \cdots$, and $X_{j}$ any vector field in the set $\left\{f^{k}, g_{1}^{k-1}, \cdots, g_{m}^{k-1}\right\}$. In a similar way we define the distributions $C_{i}(x), i=0,1, \cdots$, for $\Sigma$. Note that the distributions $C_{i}^{k}(0), i=0,1, \cdots$, defined in (3) correspond to the algorithm with $\Delta^{*}$ replaced by $T M$.

Example 3.1: Consider the nonlinear systems

$$
\Sigma: \dot{x}=g_{1}(x) u_{1}+g_{2}(x) u_{2}, \quad \Sigma^{2}: \dot{x}=g_{1}^{1}(x) u_{1}+g_{2}^{1}(x) u_{2}
$$

with $g_{1}(x)=\left(1+x_{2}, 1,1,0,1\right)^{T}, g_{2}(x)=\left(0,1+x_{3}, 0,1, x_{5}^{2}\right)^{T}$, $g_{1}^{1}(x)=\left(1+x_{2}, 1,1,0,1\right)^{T}$ and $g_{2}^{1}(x)=\left(0,1+x_{3}, 0,1,0\right)^{T}$. For $\Sigma^{2}$ and $\Sigma$ as above we readily have $C^{2}(0) \neq C(0)$. This means that neither $C^{2}(0) \subset C(0)$ or $C(0) \subset C^{2}(0)$ are true.

An explanation of the underlying idea in Example 3.1 is in order. Consider the systems $\Sigma\left(\Sigma^{k}\right)$ and the set of vector fields associated to them $\left\{f, g_{1}, \cdots, g_{m}\right\}\left(\left\{f^{k}, g_{1}^{k-1}, \cdots, g_{m}^{k-1}\right\}\right)$. It is shown that for every Lie bracket up to order $k-1$ of the vector fields $f, g_{1}, \cdots, g_{m}$ there exists a Lie bracket of the same order with the vector fields $f^{k}, g_{1}^{k-1}, \cdots, g_{m}^{k-1}$ such that they agree when evaluated at the equilibrium. This is a rough statement of what is claimed in Proposition 3.2. From this proposition a fundamental conclusion is made. Suppose the system $\Sigma$ is locally strong accessible at 0 , i.e., $\operatorname{dim} C(0)=n$. Then there exist an integer $s^{*} \geq 0$, such that the Lie brackets up to order $s^{*}$ of the vector fields $f, g_{1}, \cdots, g_{m}$ span $C(0)$. Thus, it suffices to take the $\left(s^{*}+1\right)$ th Taylor series approximation of $\Sigma$ to ensure that $\Sigma^{s^{*}+1}$ is also locally strong accessible at 0 and that $C^{s^{*}+1}(0)=C(0)$. Even more, if the distributions $C_{i}(x), i \in \underline{m}$, are assumed to be of constant dimension then $s^{*} \leq n$. Now, if the system $\Sigma$ is not strongly accessible at 0 there does not exist, in general, a relation between $C(0)$ and $C^{k}(0)$ for any $k \geq 0$.

Observe in Example 3.1 that $\Sigma$ is not locally strong accessible at 0 . Only the third-order approximation of $\Sigma$ is such that $C(0)=C^{3}(0)$ because the system $\Sigma$ equals its third-order approximation.

The next result relates the distributions $C_{i}^{k}(x)$ with $C_{i}(x)$ for $i=0,1, \cdots, k-1$. Abusing of notation, for any distribution $D(x),(D(x))^{k}$, denotes the $k$ th order Taylor series approximation of every vector field $X(x) \in D(x)$. Similarly, for $X(x) \in D(x)$, we let $(X(x))^{k}$ be the $k$ th order approximation of $X(x)$ around the equilibrium.

Proposition 3.2: Consider the analytic systems $\Sigma, \Sigma^{k}$, for a fixed $k \geq 1$ and the distributions $C_{i}, C_{i}^{k}, i=0,1, \cdots$, as defined in (3). Then

$$
\left(C_{i}^{k}(x)\right)^{k-1-i}=\left(C_{i}(x)\right)^{k-1-i}, \quad i=0,1, \cdots, k-1 .
$$

That is, for each $X(x) \in C_{i}(x)$ there exist a $\tilde{X}(x) \in C_{i}^{k}(x)$ such that $(X)^{k-1-i}=(\tilde{X})^{k-1-i}$, and vice versa.

Proof: See [9], [12]. From Proposition 3.2 it follows that $C_{i}^{k}(0)=C_{i}(0), i=0, \cdots, k-1$. Although in general $C^{k}(0) \neq$ $C(0)$ for an arbitrary $k \geq 1$, it is desirable to know if there exists a nonnegative integer $s^{*}$ for which $C^{s^{*}}(x)$, the accessibility distribution associated with $\Sigma^{s^{*}}$, yields $C^{s^{*}}(0)=C(0)$. An answer to this question is given in

Corollary 3.3: Consider the nonlinear system $\Sigma$. Assume $\Sigma$ is locally strong accessible at 0 and let $\tau$ be the smallest nonnegative integer for which $\operatorname{dim} C_{\tau}(0)=\operatorname{dim} C(0)=n$, on $M$. Then $C^{s^{*}}(0)=C(0), s^{*}=\tau+1$, with $C^{s^{*}}(x)$ the accessibility distribution associated with $\Sigma^{s^{*}}$.

The concept of accessibility distribution is closely related to that of maximal controllability distribution contained in the kernel of the output map. In the next section we define similar objects for $\Sigma$ and $\Sigma^{k}$ and find a relation between them.

\section{Controllability Distributions Contained IN THE KERNEL OF THE OUTPUT MAP DEFINED FOR $\Sigma$ AND $\Sigma^{k}$}

In this section we investigate the relation at the equilibrium, if any, of the maximal controllability distribution contained in the kernel of the output map defined for $\Sigma$ with the analogous object defined for $\Sigma^{k}$. By means of an example we exploit the importance of knowing such a relation. To some extent, a controllability distribution $\Pi$ in the kernel of the output map can be seen as the nonlinear analogous of a controllability subspace $R$ contained in the kernel of the output of a linear system of the form $\Sigma^{1}$, see [19] for some further explanation. In particular, we study the case when for a nonlinear system and the linear approximation of it the triangular decoupling problem (TDP) is solvable and compare the solutions obtained from the linearization with the solutions of the original nonlinear problem. This comparison is based on specific controllability distributions defined for both systems. Therefore we briefly review the solution of the TDP.

Given the system $\Sigma$ with $m$ outputs the problem consists in finding a regular static state feedback as defined in (1) such that 
the closed-loop system $\Sigma$ together with (1) is triangularly decoupled. That is, $v_{1}$ affects $y_{1}$ and possibly $y_{2}, \cdots, y_{m} ; v_{2}$ influences $y_{2}$ and possibly $y_{3}, \cdots, y_{m}$ but not $y_{1}$, etc. (see [7], [10], for details).

Necessary and sufficient conditions to solve the TDP for $\Sigma$ are given in terms of $\left(\Delta^{r}\right)^{*}$, the maximal controlled invariant distribution contained in

$$
\bigcap_{i=1}^{r} \operatorname{ker} d h_{i}, \quad r=0,1, \cdots, m
$$

with $\left(\Delta^{0}\right)^{*}:=C$, the accessibility distribution of $\Sigma$.

Theorem 4.1 ([16]): Assume the system $\Sigma$ satisfies $\operatorname{dim} d h(C(x))=m$, for all $x \in M$, and suppose that $\left(\Delta^{r}\right)^{*},\left(\Delta^{r}\right)^{*} \cap G$, with $\left(\Delta^{r}\right)^{*}$ the largest controlled invariant distribution in (4), have constant dimension for $r=0,1, \cdots, m$. Then the TDP is locally solvable on $M$ if and only if $\operatorname{dim}\left(\left(\Delta^{r}\right)^{*} \cap G\right)=m-r, r=0,1, \cdots, m$.

These facts are used in Example 4.2 below.

Example 4.2 ([10]): Consider the nonlinear control system

$$
\begin{gathered}
\dot{x}_{1}=u_{1}+x_{1}^{2}, \quad y_{1}=x_{1}, \\
\dot{x}_{2}=u_{1}+e^{-x_{2}} u_{2}, \quad y_{2}=x_{2} . \\
\dot{x}_{3}=u_{1}+e^{x_{2}}-x_{2}-1+x_{1},
\end{gathered}
$$

The control objective is to solve the TDP for (5). It can be checked that the conditions stated in Theorem 4.1 are satisfied and thus the TDP is locally solvable for (5).

The linearization of (5) around $\left(x_{0}, u_{0}\right)=(0,0)$ is given by

$$
\begin{gathered}
\dot{z}_{1}=\tilde{u}_{1}, \quad \tilde{y}_{1}=z_{1}, \\
\dot{z}_{2}=\tilde{u}_{1}+\tilde{u}_{2}, \quad \tilde{y}_{2}=z_{2} . \\
\dot{z}_{3}=\tilde{u}_{1}+z_{1},
\end{gathered}
$$

The solutions for the linear TDP are characterized in terms of the maximal controllability distribution contained in the kernel of $d \tilde{y}_{1}$ denoted as $\tilde{R}_{2}^{*}$ and given by

$$
\tilde{R}_{2}^{*}=\operatorname{span}\left\{\left(\begin{array}{lll}
0 & 1 & 0
\end{array}\right)^{T}\right\} .
$$

Any feedback law $\tilde{u}=F z+G \tilde{v}$ which leaves the system (6) triangularly decoupled is of the form

$$
\left(\begin{array}{l}
\tilde{u}_{1} \\
\tilde{u}_{2}
\end{array}\right)=\left(\begin{array}{ccc}
f_{11} & 0 & f_{13} \\
f_{21} & f_{22} & f_{23}
\end{array}\right)\left(\begin{array}{l}
z_{1} \\
z_{2} \\
z_{3}
\end{array}\right)+\left(\begin{array}{cc}
g_{11} & 0 \\
g_{21} & g_{22}
\end{array}\right)\left(\begin{array}{l}
\tilde{v}_{1} \\
\tilde{v}_{2}
\end{array}\right) .
$$

with $g_{11} \neq 0, g_{22} \neq 0$. For the nonlinear system (5) it can be shown that $\Pi_{2}^{*}(x)=\operatorname{span}\left\{\left(\partial / \partial x_{2}\right),\left(\partial / \partial x_{3}\right)\right\}$, where $\Pi_{2}^{*}$ is the maximal controllability distribution contained in the kernel of $d y_{1}$. But any feedback that leaves the nonlinear system (5) triangularly decoupled must have the form

$$
\left(\begin{array}{l}
u_{1} \\
u_{2}
\end{array}\right)=\left(\begin{array}{c}
\alpha_{1}\left(x_{1}\right) \\
\alpha_{2}(x)
\end{array}\right)+\left(\begin{array}{cc}
\beta_{11}\left(x_{1}\right) & 0 \\
\beta_{21}(x) & \beta_{22}(x)
\end{array}\right)\left(\begin{array}{l}
v_{1} \\
v_{2}
\end{array}\right)
$$

with $\beta_{11}(x) \neq 0, \beta_{22}(x) \neq 0$. Hence, if $f_{13} \neq 0$ in (8) then (8) does not correspond to a linearization of (9), i.e., the linear solution is not a first order approximation of a true solution for the nonlinear TDP. Basically, the reason for which the linear feedback law (8) is not a linearization, in general, of (9) is this: for a system with $m=2$ which is triangularly decouplable any feedback of the form (1) leaving the system triangularly decoupled must satisfy [7], [10]

$$
X\left(\alpha_{1}\right)(x)=0, \quad X\left(\beta_{11}\right)(x)=0, \quad \beta_{12}(x)=0
$$

for all vector fields $X \in \Pi_{2}^{*}$. Note that the feedback (9) satisfies (10). The feedback (8) also must satisfy conditions of the same type as (10), namely

$$
\tilde{X}\left(\tilde{\alpha}_{1}\right)=0, \quad \tilde{X}\left(\tilde{\beta}_{11}\right)=0, \quad \tilde{\beta}_{12}=0
$$

for all $\tilde{X} \in \tilde{R}_{2}^{*}$, with $\tilde{\alpha}_{1}(z):=f_{1} z_{1}+f_{2} z_{2}+f_{3} z_{3}, \tilde{\beta}_{11}(z):=$ $g_{11}, \tilde{\beta}_{12}(z):=g_{12}$. But since the distributions $\tilde{R}_{2}^{*}$ and $\Pi_{2}^{*}$ do not agree at the equilibrium, (8) does not correspond to the linearization of (9). On the other hand, if in the system (5) we would have that $\dot{x}_{3}=u_{1}+x_{1}$, we obtain $\Pi_{2}^{*}(0)=\tilde{R}_{2}^{*}$. Since these distributions agree at 0 we are able to approximately solve the original local nonlinear TDP by using the linear feedback (8).

To state the main result of this section an assumption concerning $\Delta^{*}$ and $\Delta_{k}^{*}$ is made.

Assumption 3: Consider the systems $\Sigma$ and $\Sigma^{k}$. Suppose that $\Delta_{k}^{*}(0)=\Delta^{*}(0)$.

Remark 4.3: If this assumption is dropped it seems not to be possible to conclude any relation between $\Pi^{*}(0)$ and $\Pi_{k}^{*}(0)$; at least not by analysis of the algorithm. Sufficient conditions for the systems $\Sigma$ and $\Sigma^{k}$ to satisfy Assumption 3 are analogous to those given in [11] where the systems $\Sigma^{k}$ and $\Sigma^{k+1}$ were treated. Observe also that for the Example 4.2, Assumption 3 is fulfilled.

Theorem 4.4: Consider the systems $\Sigma, \Sigma^{k}, k \geq 1$ and the constant dimensional distributions $\Delta^{*}, \Delta_{k}^{*}$, together with the algorithm. If Assumptions 1-3 are satisfied then

1) $\left(\Pi_{j}^{k}(x)\right)^{k-1-j}=\left(\Pi_{j}(x)\right)^{k-1-j}, j=0,1, \cdots, k-1$,

2) $\Pi_{j}^{k}(0)=\Pi_{j}(0), j=0,1, \cdots, k-1$,

3) If $k^{*}(k)=k^{*} \leq k-1$ then $\Pi_{k}^{*}(0)=\Pi^{*}(0)$,

4) If $k^{*}(k)<k^{*}$ and $k^{*}(k) \leq k-1$ then $\Pi_{k}^{*}(0) \subseteq \Pi^{*}(0)$.

where $\Pi_{j}^{k}\left(\Pi_{j}\right)$ corresponds to the $j$ th step of the algorithm applied to $\Sigma^{k}(\Sigma)$.

Proof: See [9], [12].

\section{CONCLUSIONS}

A relation at an equilibrium point between maximal controllability distributions defined for a nonlinear system and the $k$ th order Taylor series approximation of it is given, provided particular maximal controlled invariant distributions defined for both systems agree at the equilibrium. If Assumption 1 is satisfied there exists a nonnegative integer $s^{*}$ for which locally strong accessibility of the $s^{*}$ th order Taylor series approximation of a nonlinear system implies this property on the original nonlinear system. Whenever the solutions for a nonlinear synthesis control problem are characterized in terms of maximal controllability distributions we have identified some cases in which it is possible to locally approximately solve the nonlinear problem by using a solution obtained from the associated problem for the $k$ th order Taylor series approximated system.

\section{ACKNOWLEDGMENT}

The authors thank anonymous referees for some helpful comments on an earlier version of the manuscript.

\section{REFERENCES}

[1] E. G. Gilbert, "The decoupling of multivariable systems by static state feedback," SIAM J. Contr. Optim., vol. 17, pp. 50-63, 1969.

[2] L. C. J. M. Gras, H. Nijmeijer, "Decoupling in nonlinear systems: From linearity to nonlinearity," IEEE Proc. Pt. D., vol. 136, pp. 53-62, 1989.

[3] I. J. Ha and E. G. Gilbert, "A complete characterization of decoupling control laws for a general class of nonlinear systems," IEEE Trans. Automat. Contr., vol. 31, pp. 823-830, 1986.

[4] H. J. C. Huijberts and H. Nijmeijer, "Local nonlinear model matching from linearity to nonlinearity," Automatica, vol. 26, pp. 175-183, 1990 
[5] A. Isidori, Nonlinear Control Systems, (2nd ed.). Berlin: SpringerVerlag, 1989.

[6] A. J. Krener, "Approximate linearization by state feedback and coordinate change," Syst. Contr. Lett., vol. 5, pp. 181-185, 1984.

[7] H. Nijmeijer, "The triangular decoupling problem for nonlinear control systems," Nonl. Analysis Th. Meth. Appl., vol. 8, pp. 273-279, 1984.

[8] H. Nijmeijer and A. J. van der Schaft, Nonlinear Dynamical Control Systems, New York: Springer-Verlag, 1990.

[9] A. C. Ruiz, "Nonlinear control problems and systems approximations: A geometric approach," Ph.D. dissertation, University of Twente, Enschede, The Netherlands, 1993.

[10] A. C. Ruiz and H. Nijmeijer, "Further results in the nonlinear triangular decoupling problem," in Proc. 1st European Contr. Conf. (ECC) Grenoble, France, 1991, pp. 1490-1495.

[11] _ _ "Nonlinear control and systems approximations: A geometric approach," in Proc. Nonlinear Contr. Syst. Design Symp., Bordeaux, France, June, 1992, pp. 106-111.

[12] _ , "Controllability distributions and systems approximations: A geometric approach," in Proc. 31st CDC, Tucson, Arizona, 1992, pp 90-95.

[13] E. Sontag, Mathematical Control Theory, New York: Springer-Verlag, 1990.

[14] K. Shimizu and M. Matsubara, "Decoupling and disturbance rejection control of a binary distillation column with pressure variation," $J$. Chem. Eng. Japan, vol. 17, pp. 262-269, 1984.

[15] _ "Triangular decoupling for a binary distillation column under pressure control," IEEE Trans. Automat. Contr, vol. 30, pp. 474-477, 1985

[16] H. J. Sussmann and V. Jurdjevic, "Controllability of nonlinear systems," J. Differential Equations, vol. 12, pp. 95-116, 1972.

[17] T. Takamatsu, I. Hashimoto, and Y. Nakai, "A geometric approach to multivariable control systems design of a distillation column," Automat ica, vol. 15 , pp. $387-402,1979$.

[18] A. J. van der Schaft, "Relation between $H_{\infty}$ optimal control of a nonlinear system and its linearization," in Proc. 30-th $C D C$, Brighton, England, 1991, pp. 1807-1809.

[19] L. L. M. Van der Wegen, Local Disturbance Decoupling with Stability for Nonlinear Systems, (Lecture Notes in Control Information Science) vol. 166. Berlin: Springer-Verlag, 1991.

[20] W. M. Wonham, Linear Multivariable Control: A Geometric Approach, (3rd ed.). New York: Springer-Verlag, 1985

\section{A Recursive Schur-Based Solution of the Four-Block Problem}

Tiberiu Constantinescu, Ali H. Sayed, and Thomas Kailath

Abstract-We describe a new solution to the four-block problen using the method of generalized Schur analysis. We first reduce the general problem to a simpler one by invoking a coprime factorization with a block-diagonal inner matrix. Then, using convenient spectral factorizations, we are able to parameterize the unknown entry in terms of a Schur-type matrix function, which is shown to satisfy a finite number of interpolation conditions of the Hermite-Féjer type. All possible interpolating functions are then determined via a simple recursive procedure that constructs a transmission-line (or lattice) cascade of elementary $J$ lossless sections. This also leads to a parameterization of all solutions of the four-block problem in terms of a linear fractional transformation.

\section{INTRODUCTION}

A central problem in $H^{\infty}$-optimal control is the design of stabilizing controllers that minimize or at least impose an upper bound on the $H^{\infty}$-norm of the closed-loop transfer function. This problem has been widely studied in the literature and we may refer to the monograph of Francis [1] and the notes of Doyle [2] for more details and discussion. The existing approaches cover a wide range of settings and contexts. Doyle and Francis [2], [3] reduced the equivalent so-called model matching problem to a one-block (or Nehari) problem, which was then solved using the theory studied by Ball and Helton [4]. Foias and Tannenbaum [5] approached the four-block distance problem within the framework of skew Toeplitz operators and studied the associated spectral properties. Ball and Cohen [6] gave a parameterization of all suboptimal solutions based on $J$-spectral factorization theory, while Kimura and Kawatani [7] employed the notion of conjugation. Doyle et al. [8] provided state-space formulas for the stabilizing controllers by employing a separation argument and replacing the four-block problem by a pair of two-block problems. Most recently, Glover et al. [9] (see also Limebeer et al. [11], [10]) described a state-space procedure that yields an all-pass dilation of the original problem; part of this all-pass matrix was shown to generate all solutions.

We present a new solution that approaches the four-block problem within the framework of generalized Schur analysis and leads to a transmission-line (or lattice) structure that parameterizes all possible unknown entries. The derivation can be summarized as follows: we use a special factorization, with a block-diagonal inner factor, that reduces the original four-block problem with $L^{\infty}$ functions to an equivalent problem with $H^{\infty}$ functions. We then invoke convenient spectral factorizations and an inner dilation to express all possible choices of the unknown entry in terms of a Schur matrix function, which is shown to be characterized by a finite number

Manuscript received March 12, 1992; revised March 9, 1993. This research was supported in part by the Air Force Office of Scientific Research, Air Force Systems Command under Contract AFOSR91-0060, by the Army Research Office under Contract DAAL03-89-K-O109, and by a fellowship (for A. H. Sayed) from Fundação de Amparo à Pesquisa do Estado de São Paulo while on leave from the Department of Electrical Engineering, Escola Politećnica da Universidade de São Paulo, Brzail.

T. Constantinescu is with Programs in Mathematical Sciences, University of Texas at Dallas, Richardson, TX 75083 USA.

A. H. Sayed is with the Department of Electrical and Computer Engineering, University of California, Santa Barbara, CA 93106 USA.

T. Kailath is with the Information Systems Laboratory, Stanford University, CA 94305 USA

IEEE Log Number 9401670. 